\title{
Effects of exogenous adiponectin supplementation in early pregnant PCOS mice on the metabolic syndrome of adult female offspring
}

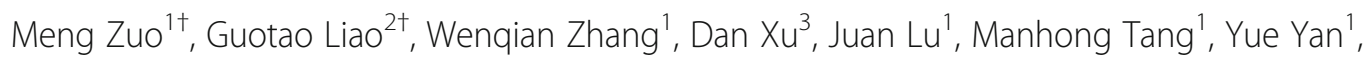
Chenghao Hong ${ }^{1}$ and Yuxia Wang ${ }^{1 *}$

\begin{abstract}
Objective: PCOS is a heterogeneous endocrine disorder with both reproductive and metabolic abnormalities. At present, PCOS has been confirmed to have a certain genetic background. Compared with healthy women, the vast majority of PCOS patients have hyperandrogenemia, and this excessive androgen exposure during pregnancy may affect the development of female fetuses. The aim of the current study was to investigate the effect of adiponectin intervention during early pregnancy of obese mice with PCOS on the metabolic phenotype of adult female offspring.

Methods: After the PCOS model was established, C57BL/6J mice were divided into maternal-control, maternalPCOS, and maternal-PCOS + APN groups. DHEA-induced PCOS mice were supplemented with adiponectin (10 mg/ $\mathrm{kg} /$ day) in the early pregnancy in order to eliminate adverse hormone exposure and then traced for endocrine indicators in their adult female offspring, which were observed for metabolism syndrome or endocrine disturbance and exhibited the main effects of APN. To further explore the underlying mechanism, the relative expressions of phosphorylated AMPK, PI3K, and Akt were detected in the ovaries of offspring mice.

(Continued on next page)
\end{abstract}

\footnotetext{
*Correspondence: sunneywang@126.com

Meng Zuo and Guotao Liao equal contributors.

'Department of Reproductive Medicine, The First Affiliated Hospital, Jinan

University, 601 West Huangpu Avenue, 510000 Guangzhou, People's

Republic of China

Full list of author information is available at the end of the article
}

(c) The Author(s). 2021 Open Access This article is licensed under a Creative Commons Attribution 4.0 International License, which permits use, sharing, adaptation, distribution and reproduction in any medium or format, as long as you give appropriate credit to the original author(s) and the source, provide a link to the Creative Commons licence, and indicate if changes were made. The images or other third party material in this article are included in the article's Creative Commons licence, unless indicated otherwise in a credit line to the material. If material is not included in the article's Creative Commons licence and your intended use is not permitted by statutory regulation or exceeds the permitted use, you will need to obtain permission directly from the copyright holder. To view a copy of this licence, visit http://creativecommons.org/licenses/by/4.0/ The Creative Commons Public Domain Dedication waiver (http://creativecommons.org/publicdomain/zero/1.0/) applies to the data made available in this article, unless otherwise stated in a credit line to the data. 


\begin{abstract}
(Continued from previous page)
Results: The serum testosterone level of the maternal-PCOS + APN group in early pregnancy was significantly lower than that of the maternal-PCOS group $(p<0.01)$. The serum testosterone level in the offspring-PCOS + APN group was significantly lower than in the offspring-PCOS group $(p<0.05)$, the diestrus time characterized by massive granulocyte aggregation in the estrus cycle was significantly shorter than in the offspring-PCOS group $(p<0.05)$, and the phenotypes of PCOS-like reproductive disorders and metabolic disorders, such as obesity, insulin resistance, impaired glucose tolerance, and hyperlipidemia, were also significantly improved in the offspring-PCOS + APN group $(p<0.05)$. Compared with the control group, the expression levels of phosphorylated AMPK, PI3K, and Akt in the offspring-PCOS group were significantly decreased $(p<0.05)$, while those in the offspring-PCOS + APN group were significantly increased $(p<0.05)$.

Conclusions: APN intervention in early pregnancy significantly reduced the adverse effects of maternal obesity and high androgen levels during pregnancy on female offspring and corrected the PCOS-like endocrine phenotype and metabolic disorders of adult female offspring. This effect may be caused by the activation of the AMPK/PI3K-Akt signaling pathway in PCOS offspring mice.
\end{abstract}

Keywords: Adiponectin, Polycystic ovary syndrome, Offspring, Metabolic syndrome

\section{Background}

Polycystic ovary syndrome (PCOS) is one of the most common endocrine diseases in women of childbearing age with a prevalence rate of $10-18 \%$ [1, 2]. Clinically, it is characterized by hyperandrogenism, ovulatory dysfunction, and polycystic ovary morphology, often accompanied by insulin resistance and obesity [3-5]. Moreover, prevalence rates increase to over $25 \%$ in severely obese women with PCOS [6]. The etiology of PCOS has not been clarified yet, but, in recent years, more and more attention has been paid to the role of environmental and genetic factors in the occurrence and development of PCOS [7]. Family aggregation and twin studies have shown that PCOS has a strong heritable component [8]. However, the gene mutations identified so far cannot explain the high prevalence of PCOS in the population, which means that fetal exposure to environmental factors may also play an important role in the pathogenesis of this disease [9]. The interaction between heredity and sex hormones jointly affects the development of sexual dimorphism in mammalian offspring, while the poor nutritional status and inappropriate hormone levels of PCOS mothers during pregnancy may lead to metabolic disorders in offspring and permanently change the related endocrine phenotype of adult offspring [10-12]. Studies have pointed out that the exposure to adverse sex hormones during pregnancy and shortly after birth may affect the metabolism in adulthood. For such offspring of adult female rats, the testosterone marker showed obvious insulin resistance and metabolic dysfunction [13]. Eliminating the influence of inappropriate testosterone in the sensitive period is expected to be the key to normalize the endocrine phenotype of the offspring.

Adiponectin (APN) is a highly specific $30 \mathrm{kDa}$ protein expressed by adipose tissue [14] and exists in human plasma at a high concentration $(5-15 \mathrm{ug} / \mathrm{mL}$, accounting for $0.01 \%$ of the total plasma protein) [15]. APN plays an important role in inhibiting the growth and metastasis of tumor cells because of its anti-proliferation effect and induction of apoptosis [16, 17]. In addition, APN improves the insulin sensitivity, is anti-inflammatory, and can participate in the prevention and treatment of diabetes and atherosclerosis $[18,19]$. Insulin sensitivity is improved by APN through the cross-linking of multiple pathways. APN can induce extracellular calcium influx by activating AdipoR1 [20], which leads to the direct phosphorylation of AMP-activated protein kinase (AMPK) by calcium/calmodulin dependent protein kinase kinase2 (CAMKK2) in Thr172 [21, 22]. AMPK activated by APN can directly inhibit ribosomal protein S6 kinase 1 (S6K1); on the one hand, it reduces the risk of insulin resistance caused by increased phosphorylation of insulin receptor substrate-1 (IRS-1) in Ser636/639 [23], and, on the other hand, AMPK can also activate the phosphatidylinositol 3-kinase/protein kinase B (PI3K/Akt) signal transduction pathway for insulin signal transmission and regulation of the glucose metabolism [22, 24]. In addition, Akt can phosphorylate its own Ser485 or intermediate glycogen synthase kinase $3 \beta$ (GSK3ß) for negative feedback regulation of AMPK [25].

Some studies have shown that under the regulation of pregnancy-related molecules, the level of adiponectin in pregnancy is significantly higher than in non-pregnancy, while female patients with overweight or PCOS show generally a lower level of adiponectin than normal women [26], and they are more likely to suffer from metabolic diseases, such as gestational diabetes mellitus, during pregnancy $[27,28]$. Based on the observation of the pregnant mouse model of APN deficiency, Qiao et al. found that pregnant mice with APN gene (Adipoq $^{-/}$) knockout showed abnormal glucose 
tolerance and hyperlipidemia in the third trimester of pregnancy, accompanied by fetal weight gain and abnormal offspring metabolism [29]. It is worth noting that these defects can be eliminated by adenovirus-mediated APN gene reconstruction during pregnancy to finally improve the quality of islet $B$ cells.

Based on the important role of APN in regulating fatty acid oxidation and improving insulin resistance and the vertical effect of PCOS maternal endocrine environment on female offspring, this study focused on female offspring of obese mice with PCOS by observing the relevant metabolic phenotypes of each offspring after reaching adulthood to analyze the effect of APN supplementation during early embryo development on PCOSrelated metabolic disorders in adult female offspring and further explore its mechanism.

\section{Methods}

\section{Animal maintenance}

All experimental procedures were approved by the Animal Ethics Committee of Jinan University. Immature female wild-type C57BL/6J mice $(n=60$, four weeks old, $13 \pm 1 \mathrm{~g}$ ) were purchased from the Centre for Experimental Animals (Shandong, China). The animals were group-housed under standard laboratory conditions (room temperature: $22.5 \pm 2.5{ }^{\circ} \mathrm{C}$; relative humidity: $45.0 \pm 2.0 \%$; $12 \mathrm{~h}$ light/dark cycle with lights on at 8:00 am). Tap water and food pellets were provided ad libitum. The mice were kept for one week to acclimatize to the conditions and weighed daily during adaptive feeding and modeling.

Female mice were initially divided into (PCOS) model $(n=45)$ and control groups $(n=15)$. For modeling, the model group were given a high-fat diet (HFD: $60 \%$ fat content, China) [30] and subcutaneously injected with $0.6 \mathrm{mg} / \mathrm{kg} /$ day dehydroepiandrosterone (DHEA: Coolober Technology Co., Ltd., Beijing, China) [31] dissolved in $0.2 \mathrm{~mL}$ soy oil for 21 consecutive days. The control group were given a standard diet (SD:12 mm; Breeding Food, China) and subcutaneously injected with the same dose of $0.9 \%$ sodium chloride. After modeling, three mice in each group were randomly selected to verify successful modeling by the trends of body weight, serum testosterone level, and ovarian tissue section.

\section{Exogenous APN treatment in early pregnancy}

After successful modeling, the model group $(n=39$, three mice dead, three mice sacrificed) were randomly divided into two groups: maternal-PCOS group $(n=20$; $0.9 \%$ sodium chloride, s.c.) and maternal-PCOS + APN group ( $n=19$; APN, $10 \mathrm{mg} / \mathrm{kg} /$ day, s.c.) [32]. The control group was recorded as maternal-control group $(n=$ 10 , two mice dead, three mice sacrificed; $0.9 \%$ sodium chloride, s.c.). Female mice received 10 IU pregnant mare serum gonadotropin (PMSG: ProSpec, Inc., Israel) and were injected with 10 IU human chorionic gonadotropin (HCG: LIVZON, Inc., China) 48 h later. Females of the different groups were randomly mated with untreated C57BL/6J wild-type males (8 weeks old, $25 \pm$ $2 \mathrm{~g})$. Mice with vaginal plug were recorded at day 0 of pregnancy.

Adiponectin (APN: gAcrp30, murine recombinant, BioVision, USA) was dissolved in Tris solution ( $1 \mathrm{~mL}$, $5 \mathrm{mM}, \mathrm{pH}$ 7.6), and this stock solution was used to prepare an APN solution with a concentration of $0.1 \mathrm{mg} /$ $\mathrm{mL}$. APN injection with this solution began on day three after pregnancy, and treatment of the maternal-PCOS + APN group was continued for 11 days. After completion of the injection, three female mice in each group were randomly selected to obtain blood samples for the detection of the levels of serum androgen and adiponectin.

The pregnancy rate was quantified per treatment and pairing throughout the observation process. Pregnant mice were assigned to the corresponding groups at the

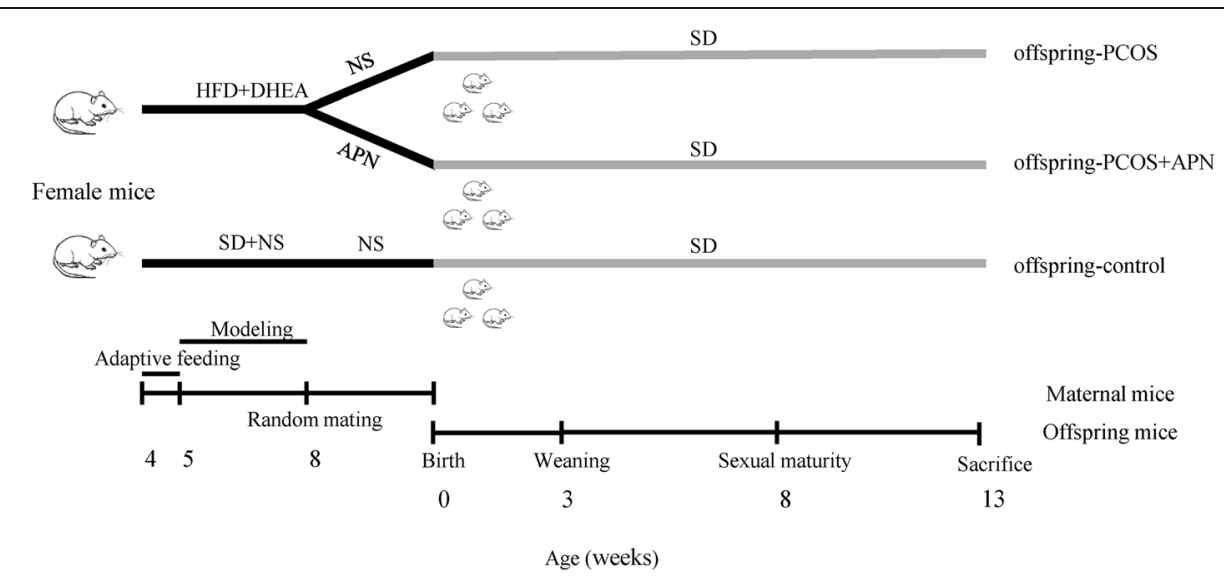

Fig. 1 Schematic representation of the different experimental groups: offspring-control group ( $n=6$; maternal-control group offspring, SD); offspring-PCOS group ( $n=6$; maternal-PCOS group offspring, SD); offspring-PCOS + APN group $(n=6$; maternal-PCOS + APN group offspring, SD) 
Table 1 Fertility indexes of mice of each group (mean \pm SD)

\begin{tabular}{lcccc}
\hline Groups & $\begin{array}{l}\text { Total } \\
\text { (pcs) }\end{array}$ & $\begin{array}{l}\text { Pregnancy } \\
\text { (pcs) }\end{array}$ & $\begin{array}{l}\text { Pregnancy rate } \\
\text { (\%) }\end{array}$ & $\begin{array}{l}\text { Average litter size } \\
\text { (pcs) }\end{array}$ \\
\hline maternal-control & 7 & 6 & 85.71 & $7.00 \pm 2.28$ \\
maternal-PCOS & 17 & 5 & $29.41^{*,++}$ & $1.80 \pm 0.30^{* *},+$ \\
maternal-APN + PCOS & 16 & 13 & 81.25 & $4.08 \pm 2.18^{*}$ \\
\hline
\end{tabular}

${ }^{*},{ }^{* *}$ indicate significance of the maternal-control group at $p<0.05$ and $p<0.01$ probability level, respectively; ${ }^{+}{ }^{++}$indicate significance of the maternal-PCOS + APN group at $p<0.05$ and $p<0.01$ probability level, respectively

time of weaning (Fig. 1). The standard diet was given to all mice during breeding, lactation, and growth of the young stock. The nutritional profile of the standard diet was the following: protein $20 \%$, fat $4 \%$, fiber $5 \%$, ash $8 \%$, moisture 10\%, nitrogen-free extract 50\%; calories: $3.78 \mathrm{kcal} / \mathrm{g}$.

\section{Phenotypic indices and vaginal cytology}

Bodyweight (BW) and food intake of offspring mice were monitored daily from weaning (3 weeks old) to slaughter (13 weeks old). In adulthood (8 weeks old), vaginal smears were performed daily for 20 consecutive days (4-5 cycles) [33]. The estrous cycle of offspring mice was monitored by vaginal smears and assessed by optical microscopy.

\section{I.p. Glucose Tolerance Test (IPGTT) and I.p. Insulin Tolerance Test (IPITT)}

IPGTTs were performed of non-fasted offspring mice (8 weeks old), and their food was removed $16 \mathrm{~h}$ before the experiment. One week after IPGTT, IPITTs were performed of mice that were fasted for $6 \mathrm{~h}$. Tests were performed by i.p. injecting mice with either glucose or insulin, as previously described (IPGTT: 10\% glucose, $0.5 \mathrm{~g} / \mathrm{kg}$ BW; IPITT: insulin $2 \mathrm{U} / \mathrm{kg}$ BW). The blood glucose concentration of tail vein blood was measured with a glucose monitoring kit (Johnson, USA) at 0, 15, 30, 60, 90 , and $120 \mathrm{~min}$ after glucose or insulin injection.

\section{Tissue and blood sample collection}

At the end of the experiment, all offspring mice (13 weeks old) were anesthetized, blood samples were obtained by eyeball enucleation, and food was removed overnight for a maximum of $8 \mathrm{~h}$ (water was unlimited) before slaughter. The serum was immediately separated and stored at $-20{ }^{\circ} \mathrm{C}$ for subsequent analyses. After collection of blood samples, the bilateral ovaries of the mice were rapidly removed, and some of the fresh ovarian tissue samples were rapidly frozen at $-80{ }^{\circ} \mathrm{C}$ for biochemical analysis. The other part of the ovaries were fixed in $4 \%$ paraformaldehyde solution. After embedding in paraffin, the ovaries were cut into $5-\mu \mathrm{m}$ thick sections and stained with hematoxylin-eosin $(\mathrm{H} \& \mathrm{E})$, and the tissue structure was observed by electron microscopy.
Biochemical analyses and serum hormone measurements Fasting blood glucose (FBG), total cholesterol (TC), triglycerides (TG), HDL cholesterol (HDL-C), and LDL cholesterol (LDL-C) levels in the serum were measured using an autoanalyzer (BS-5800M, Mindray, China). The homeostasis model assessment-insulin resistance (HOMA-IR) index was calculated as previously described [34]. Serum insulin (INS), adiponectin (APN), and testosterone $(\mathrm{T})$ concentrations were measured using an ELISA kit (CUSABIO, Wuhan, China) according to the manufacturer's instructions.

\section{RNA extraction and real-time PCR}

Total RNA was extracted from the ovarian tissue of offspring mice with the TRIzol reagent (Invitrogen) to synthesize first-strand cDNAs by using the EasyScript TM First-strand cDNA Synthesis SuperMix (TransGen Biotech, China). Quantitative real-time PCR (qRT-PCR) was performed to determine the total CDNA with a 7500 real-time PCR system (Applied Biosystems, USA). The qRT-PCR was performed using a ImProm-II ${ }^{\mathrm{TM}}$ Reverse Transcription System (Promega, USA). Specific primers for amplification of specific genes were purchased from Sangon Biotech (Shanghai, China), as listed in Table 1. Expression levels were determined by the $2^{-\Delta \Delta C t}$ method, Cicated as the ratio with respect to the control.

\section{RT- PCR primer sequences}

\begin{tabular}{ll}
\hline Primer name & Nucleotide sequence (5'-3') \\
AKT- forward & CAAGGCCCAACACCTTATC \\
AKT- reverse & ACGATGACCTCCTTCTTGAG \\
AMPK- forward & TGTAGAGCAATCAAGCAGTT \\
AMPK- reverse & TCCTTTGGCAAGATCGATAG \\
PI3K- forward & GGGCAGTTAAGAAGCACAATG \\
PI3K- reverse & GCAGGAGAGTCTTTCCAATG \\
GAPDH- forward & GGCCTCCAAGGAGTAAGAAA \\
GAPDH- reverse & GCCCCTCCTGTTATTATGG \\
\hline
\end{tabular}

\section{Western blotting}

Protein was extracted from ovarian tissue of offspring mice. Total protein concentrations were measured using 
a BCA protein assay kit (Solarbio, China). Equal amounts of proteins $(15 \mu \mathrm{g})$ were resolved by SDSPAGE and then electrophoretically transferred to PVDF membranes. Subsequently, the membrane was blocked with 5\% BSA (Solarbio, China) for $2 \mathrm{~h}$ and probed with different primary antibodies at $4{ }^{\circ} \mathrm{C}$ overnight. The main primary antibodies were phospho-AMPK $\alpha$ (Thr172; CST, USA), phospho-PI3 kinase p85(Tyr458)/ p55(Tyr199) antibody (CST, USA), phospho-Akt (Ser473) antibody (CST, USA), phospho-Fox03a (Ser253) antibody (CST, USA), caspase-3 antibody (CST, USA), and Fox03a antibody (Abcam, UK). After thoroughly washing three times with TBST for 5 min each time, HRP-linked secondary antibody (CST, USA) was used to detect the primary antibodies, followed by an additional 1-h incubation at room temperature. At last, bands were visualized using an Amersham Imager 600 (GE, USA), and band intensities were analyzed using the computer Image J 6.0 software (National Institutes of Health, USA).

\section{Statistical analyses}

All data were expressed as the mean $\pm \mathrm{SD}$ and analyzed using the GraphPad Prism 8.0 (GraphPad Software, Inc., USA) and SPSS 25.0 (SPSS, Inc., Chicago, IL, USA). The Wilcoxon rank-sum test was used to compare two independent samples of the original data, and the KruskalWallis test was used for non-normally distributed data. Multiple-group comparisons were evaluated by one-way ANOVA with post-hoc testing. Tests were performed within the $95 \%$ confidence interval, and the significance level was set at $p<0.05$.

\section{Results}

\section{Successful establishment of PCOS model and offspring} breeding

The difference between the weight gain of mice in the model and control groups was statistically significant $(p<0.05$; Fig. 2b). The serum testosterone level in the PCOS model group was significantly higher than in the control group $(p<0.01)$, which is consistent with the

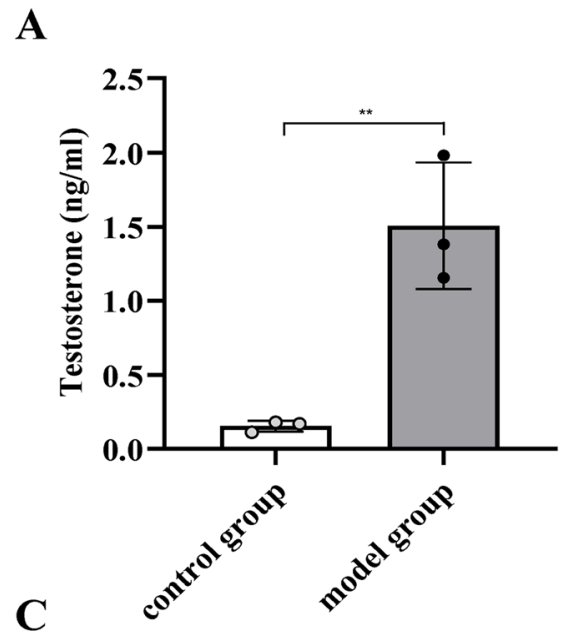

B
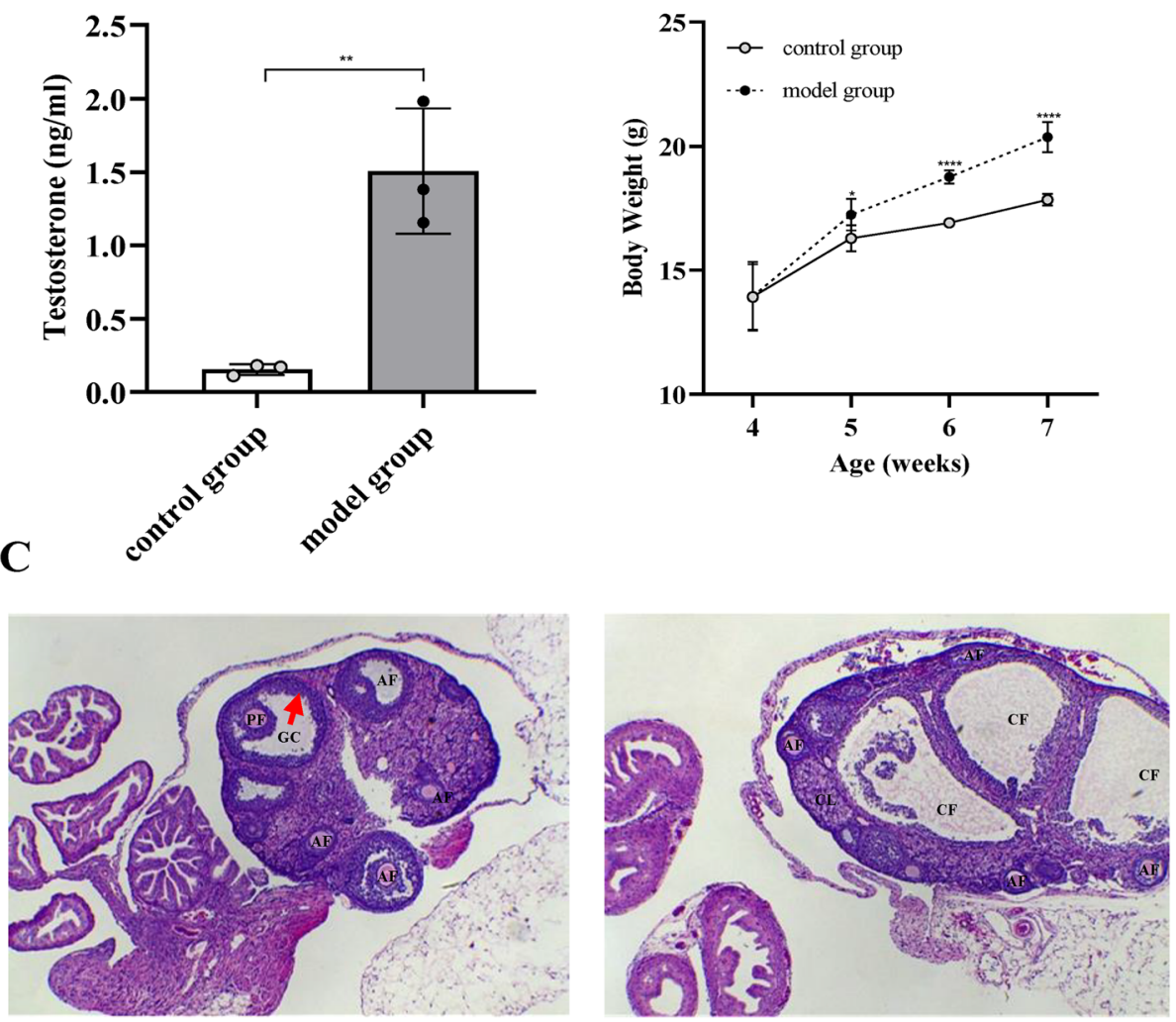

control group

model group

Fig. 2 Establishment of the PCOS model. a Changes in the active testosterone expression in mice after an effective dose of DHEA stimulation; b significant differences in the body weight between the control and model groups; c ovarian tissue sections were prepared from control or model mice for H\&E staining. Antral follicle (AF), preovulatory follicle (PF), cystic follicle (CF), granulosa cell (GC), and corpora lutea (CL) were annotated. ${ }^{*} p<0.05,{ }^{* *} p<0.01,{ }^{* * *} p<0.001,{ }^{* * * *} p<0.0001$ versus untreated control mice. Data are indicated as mean $\pm \operatorname{SD}(n=3)$ 
symptoms of hyperandrogenemia in PCOS (Fig. 2a). Atresia and cystic dilated follicles were significantly increased in the model group compared with the control group. Moreover, the radiating crown disappeared, the granulosa cell layers became loose, and their number decreased. However, multiple follicles at different developmental stages were detected in the ovaries of mice in the normal control group (Fig. 2c). This indicates that the mouse PCOS model was successfully established. In addition, PCOS adult female mice also showed impaired fertility, as indicated by significantly fewer litters, showing significant differences in the pregnancy rate $\left(\chi^{2}=11.57, p\right.$ $<0.01)$ and average litter size $(\mathrm{F}=8.85, p<0.01)$ among different groups of maternal mice (Table 1 ).

\section{Prenatal hyperandrogenic exposure triggers endocrine disorders similar to PCOS in adult female offspring}

To test whether exposure to a high androgen environment during pregnancy leads to a PCOS phenotype in adult offspring, we simulated hyperandrogenic exposure in female mice during pregnancy after establishing the PCOS model and studied endocrine and reproductive features of female offspring in adulthood.

The serum androgen level of the maternal-PCOS group was higher than that of the maternal-control group $(p<0.01)$, and the maternal-PCOS group exhibited more obvious hypoadiponectinemia than the maternal-control group $(p<0.05)$. After APN intervention in early pregnancy, hyperandrogenemia and hypoadiponectinemia of maternal-PCOS mice were significantly improved.

Moreover, PCOS offspring showed weight gain, eating disorders, hyperandrogenemia $(p<0.05$; Figs. 3 and $4 \mathrm{c}$ and a), and changes in the estrous cycle. Compared with the offspring-control group, offspring-PCOS mice rarely entered the preovulatory stage of the estrous cycle and displayed prolonged time in metestrus/diestrus (Fig. 3d). Ovarian histology of PCOS progeny showed consistent abnormalities. Compared with the offspring-control group, the number of corpus luteum was significantly reduced after ovulation, and no dominant follicles appeared (Fig. 3e). Phenotypic characterization of the offspring (Fig. 4b) showed that prenatal hyperandrogenic exposure triggers endocrine disorders similarly to PCOS in offspring mice, which seems to be effectively improved by maternal APN intervention in early pregnancy.

\section{Adiponectin therapy in early pregnancy of PCOS mice normalizes the endocrine phenotype of adult female offspring}

After successful modeling, pregnant mice of offspringcontrol and offspring-PCOS groups were subcutaneously injected with $0.9 \%$ saline, and the offspring-PCOS + APN group was treated with APN (10 mg/kg/day) during early pregnancy (P3-P13, 11 days) within the same temporal window.

Observation of the metabolisms of offspring-PCOS and offspring-PCOS + APN groups showed that the PCOS-like neuroendocrine phenotype and metabolic disorders of female offspring treated with APN during early pregnancy tend to normalize. Compared with the offspring-PCOS group, the fasting levels of blood glucose and insulin were decreased (Fig. 4b, d), impaired glucose and insulin tolerance were alleviated, and insulin sensitivity was improved in the offspringPCOS + APN group (Fig. 4c, e). In addition, compared with the offspring-PCOS group, total cholesterol and low-density lipoprotein levels in the serum of the offspring-PCOS + APN group were significantly decreased (Fig. 4f) and approached the levels of offspring-control mice. Surprisingly, the offspring showed completely opposite results at the adiponectin level compared with mother mice. Compared with APN-treated offspring, the adiponectin level of PCOS offspring was significantly higher (Fig. 3a, b).

\section{Effects of adiponectin on the activities of phosphorylated AMPK, PI3K, and Akt in the ovarian tissue of offspring mice}

Protein kinase activities in the ovaries were assessed by detecting the phosphorylated forms of AMPK, PI3K, and Akt and further determined based on changes in the mRNA of the cells to investigate whether AMPK, PI3K, and Akt might be responsible for the protective effect of adiponectin.

As shown in Fig. 5a, compared with the offspringcontrol group, subcutaneous injection of adiponectin during early pregnancy significantly increased the levels of AMPK, PI3K, and Akt phosphorylation in ovarian tissue, while the phosphorylation levels of the offspringPCOS group were significantly decreased. In addition, the mRNA content of phosphorylated AMPK, PI3K, and Akt was measured in the cells, revealing a consistent trend with changes in the protein phosphorylation levels (Fig. 5b-d). APN treatment in early pregnancy had a significant impact on the production of phosphorylated AMPK, PI3K, and Akt in the offspring's ovaries.

\section{Discussion}

Inappropriate hormone exposure during the early pregnancy, which is a sensitive period for different organizations or systems, including the central nervous system, heart, liver, and reproductive organs, can induce large metabolic alterations in adult life, and most women with PCOS have unexplained hyperandrogenic levels during pregnancy $[35,36]$. Cimino I et al. confirmed that the increased binding 


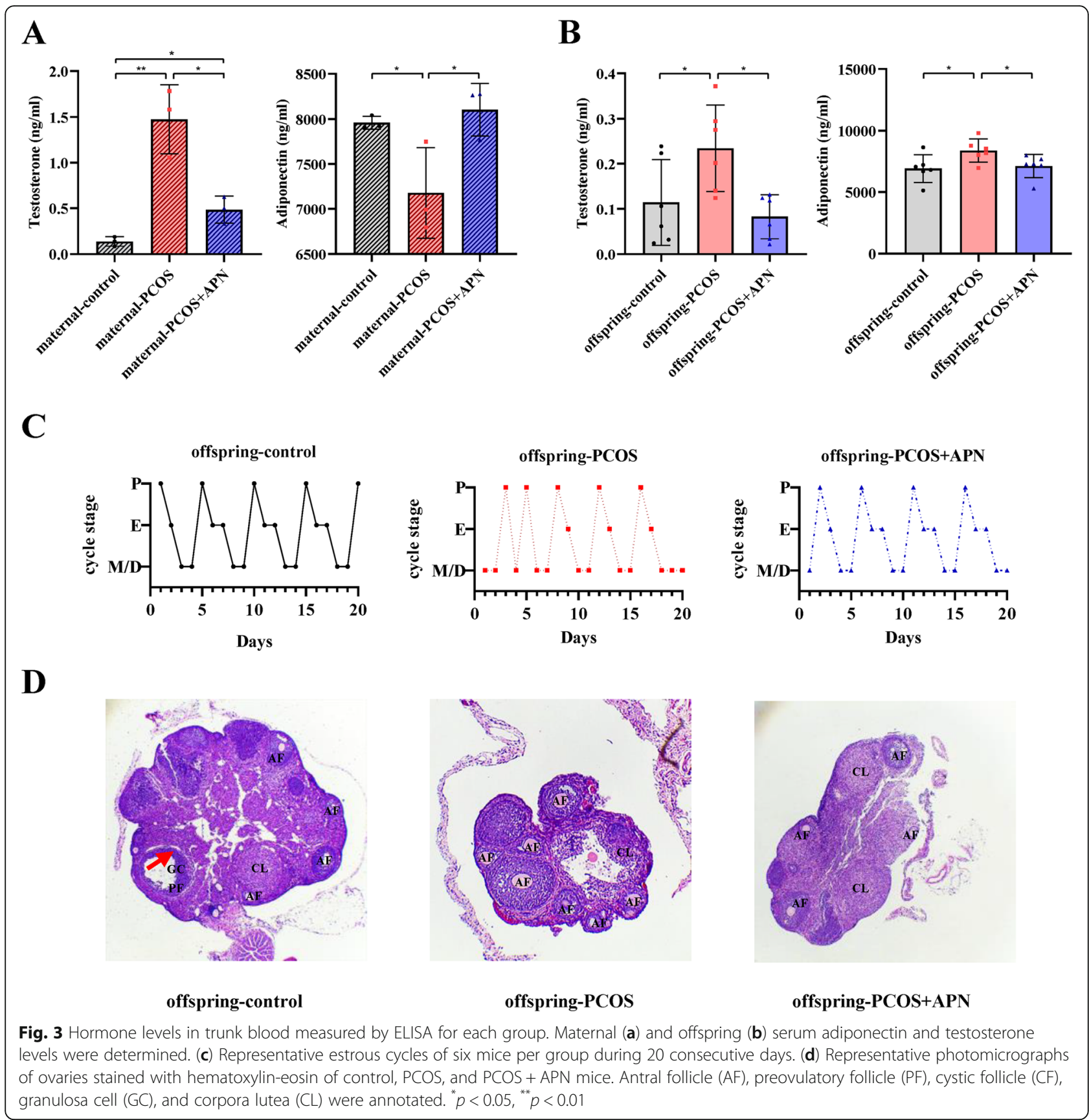

of GABA and GnRH neurons in the offspring caused by hyperandrogenemia during pregnancy. Persistent overactivity of $\mathrm{GnRH}$ neurons in the offspring after adulthood leads to great changes in HPO axis reactivity and sex hormone levels [33]. These changes activate the immune system of the offspring, which in turn leads to a chronic inflammatory response characterized by reproductive endocrine disorders [33, 37]. Meanwhile, Epidemiological analysis has demonstrated links between maternal obesity and offspring disease. The risk of organ deficits and related diseases caused by obesity has circular programming in subsequent generations [38]. In the current study, we have demonstrated that exposure to obesity and highdose testosterone during pregnancy can cause severe metabolic disorders in adult female mice of PCOS offspring, mainly manifested by hyperandrogenemia and metabolic disorders, which centered on insulin resistance. In addition, APN intervention in early pregnancy could significantly improve the hyperandrogenemia and hypoadiponectinemia of obese maternalPCOS mice, thus significantly improving the reproductive metabolic phenotype of offspring. 


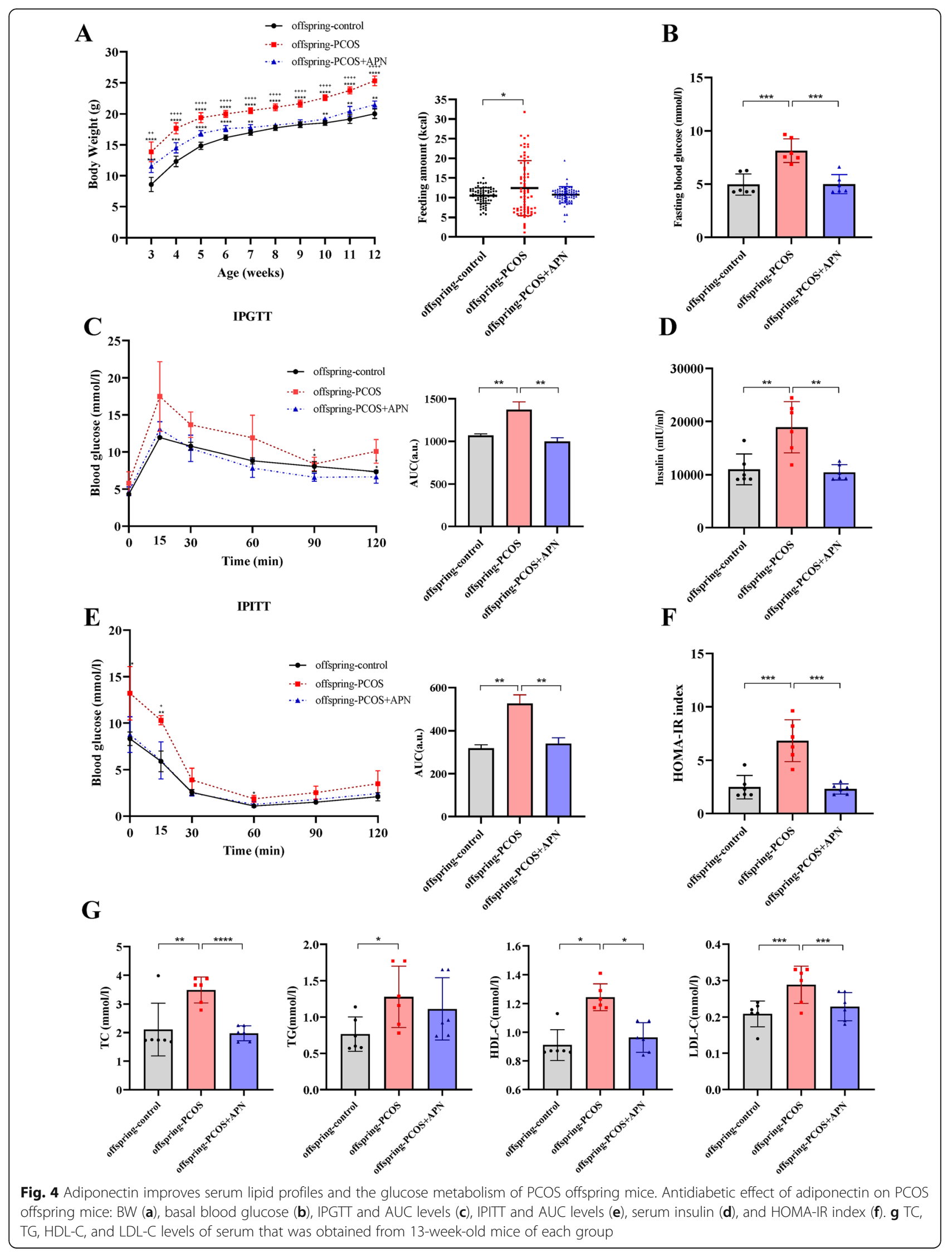



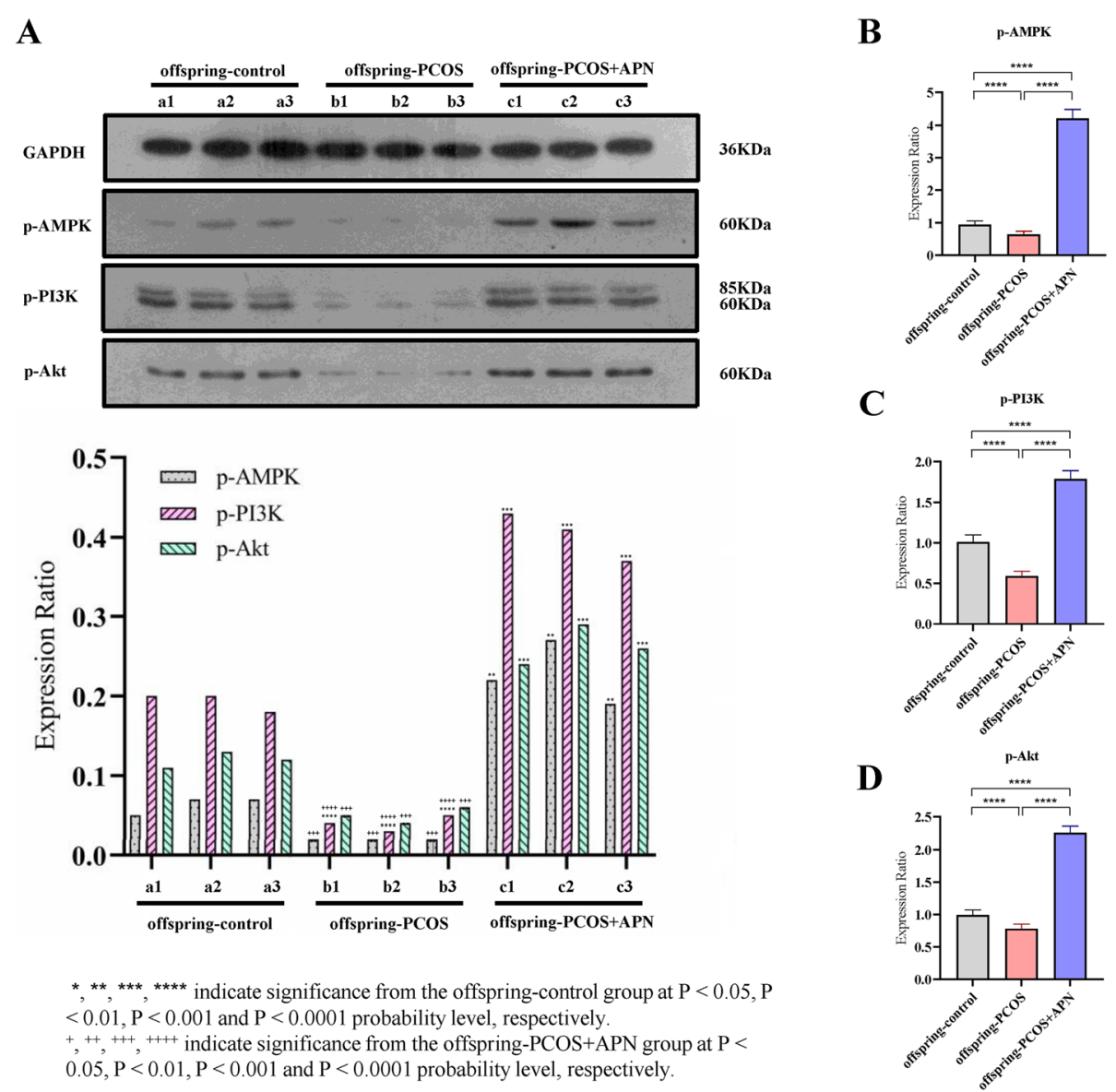

Fig. 5 Effects of adiponectin on AMPK, PI3K, and Akt phosphorylation expression in PCOS offspring mice. a Immunoblotting of protein extracts from ovarian tissues of PCOS offspring mice treated with adiponectin during the embryonic phase in comparison with control and untreated PCOS offspring mice. $\mathbf{b}$-d Content of mRNA of phosphorylated AMPK, PI3K, and Akt of mice of each group

Antenatal adverse events alone may not be sufficient to determine the occurrence of PCOS in the offspring, and adverse stimuli (endocrine imbalance and/or environmental exposure) during subsequent life after birth may reveal or amplify potential defects associated with PCOS [39]. Anderson AD et al. reported that obesity can increase the androgen production in the ovaries/adrenal glands through insulin resistance and compensatory hyperinsulinemia and inhibit sex hormone-binding globulin (SHBG) to increase the bioavailability of androgen. Both effects reinforce each other, aggravating the development of PCOS [40, 41]. Our data indicate that androgen exposure during pregnancy and severe eating habit disorders are significant factors leading to obesity in PCOS offspring. Under the premise that the average daily food intake is similar, the weight of PCOS offspring is significantly higher than that of normal offspring, and the persistent obesity during the growth of the offspring further increases the risk of hyperandrogenism in adulthood. Based on clinical observations, Sir-
Petermann et al. found that part of the metabolic characteristics of PCOS existed already in the early childhood of the daughters of PCOS patients. In contrast, the levels of serum insulin and triglyceride further increased and the level of serum SHBG decreased in the puberty of PCOS offspring, and hypoadiponectinemia occurred much earlier than hyperandrogenemia [42]. It has been confirmed that APN has a considerable degree of sensitivity and is an early sign of metabolic disorder in PCOS offspring. We found that maternal PCOS pregnant mice also showed a considerable degree of hypoadiponectinemia compared with normal pregnant mice, and the serum APN concentration of PCOS pregnant mice reached the normal level after APN supplementary intervention, which reduced the hypoadiponectinemia environmental exposure caused by hyperandrogenic and obesity of PCOS female offspring in the embryonic stage to a certain extent.

On the contrary, based on the above conclusions, we found that after adiponectin treatment, a series of 
metabolic disorders, such as insulin resistance, impaired glucose tolerance, and elevated blood lipids, were improved in PCOS offspring. In addition, the serum testosterone level of the offspring-PCOS + APN group was significantly lower than that of the offspring-PCOS group after reaching adulthood, which confirmed that the embryonic APN intervention treatment corrected the high androgen level of PCOS female offspring, reduced inflammatory reactions in the reproductive tract, and altered the estrous cycle toward a normal cycle. In addition, the weight gain of the offspring-PCOS + APN group tended to be normal during the growth process. Because of the significant effect of adiponectin treatment in early pregnancy, we believe that the adiponectin treatment process occurs in the uterus during the embryonic stage. However, the APN level in female adult mice in the offspring-PCOS group was slightly higher than that in the offspringcontrol group, which contradicts the detected serum APN levels in early pregnancy as well as prepuberty and puberty offspring, as reported by Sir-Petermann et al. [42]. Some studies have shown that pregnant women and fetuses have relatively independent adiponectin regulatory systems. Adiponectin cannot directly pass through the placental barrier, and offspring are not affected by maternal adiponectin levels [43]. This conclusion confirms that there is no correlation between the APN level of offspring mice in adulthood and that of maternal mice in early pregnancy. Some reports also confirmed that a compensatory mechanism of metabolic disorders occurs in PCOS patients, as oxidative stress markers, such as superoxide dismutase and catalase, were detected in the normal range in women with PCOS [44]. In the current study, some offspring may exhibit a series of compensatory effects, the APN level in female adult mice in the offspring-PCOS group was slightly higher than that in the offspring-control group, which is also reflected in the high-density lipoprotein cholesterol concentration in the serum of female offspring rats. We speculate that the significant effect of adiponectin treatment during pregnancy to prevent PCOS-like traits in offspring is most likely to be achieved by normalizing the maternal metabolism and reducing adverse exposure of offspring, rather than via a direct effect of adiponectin on the offspring.

In this experiment, PCOS maternal hypoadiponectinemia was effectively corrected, while PCOS maternal hyperandrogenemia was only improved to a great extent, showing that the androgen level of PCOS pregnant mice during pregnancy was still slightly higher than that of normal mothers after APN intervention. This means that offspring of female mice were still exposed to a low-dose androgen environment during the embryonic period. Studies have shown that prenatal androgen exposure has a dosedependent effect on the metabolism of offspring in adulthood [45]. On the one hand, low-dose androgen exposure can enhance the expression of steroidogenic acute regulatory protein (StAR) and peroxisome proliferator-activated receptors $\gamma$ (PPAR $\gamma$ ), which regulate the utilization of cholesterol, showing an antioxidant response in PCOS offspring; on the other hand, high-dose androgen exposure can induce a proinflammatory state in ovarian tissue mediated by a high prostaglandin level and the expression of cyclooxygenase-2 protein (COX2). In this study, we speculated that APN intervention in early pregnancy did not directly cut off the fetal androgen exposure of PCOS mice but transformed unfavorable high-dose exposure into favorable low-dose exposure, but this conjecture still needs to be verified by further experiments.

The aim of this study was to examine whether exogenous adiponectin supplementation in early pregnancy is necessary for normalizing the endocrine phenotype of adult female PCOS offspring mice. We detected the relative expressions of phosphorylated AMPK, PI3K, and Akt in the mature ovaries of PCOS offspring pretreated with or without APN during the embryonic stage and found that the AMPK/PI3K-AKT pathway was activated in the offspring-APN + PCOS group. By correlating the levels of adiponectin in each offspring, we can conclude that the AMPK-mediated PI3K/ AKT pathway is activated in adiponectin-treated offspring mice; this pathway consumes adiponectin in the circulation and decreases the adiponectin level to its normal value. However, the low expression of the AMPK/PI3KAKT pathway in PCOS offspring has undergone irreversible programming during formation in the embryonic stage $[46,47]$, and the primary dysfunction of ovarian tissue in offspring leads to the loss of its function of compensating elevated APN levels. This process leads to the accumulation of some unmetabolized APN in the peripheral circulation, resulting in a transient increase in serum APN levels. This conclusion answers the opposite result of serum adiponectin levels in the two generations of maternal and offspring, and further reflects that taking measures to eliminate adverse factors during embryogenesis is of great significance to improve the offspring's overall metabolic function. On the other hand, we can see that the offspring body's compensation mechanism for high androgen exposure during the embryonic stage is also achieved by increasing peripheral adiponectin levels, which reflects the sensitivity of APN in metabolic and reproductive regulation in PCOS patients. As a result of these data, we infer that adiponectin supplementation during the embryonic period improved the metabolic syndrome of PCOS offspring in adulthood via activation of the AMPK/PI3K-Akt pathway.

\section{Conclusions}

Our current study provided new data on the relationship between exogenous APN supplementation and the 
metabolic consequences for offspring with prolonged androgen and hyperlipidemia exposure in the embryonic period. As adiponectin works, treatment is most likely to be achieved by normalizing the metabolism of the mother and reducing the adverse exposure of the offspring. We speculated that compared with the direct treatment of metabolic disorders of PCOS offspring in adulthood, more offspring may benefit from early pregnancy treatment. This is supported by the fact that for PCOS patients with abnormal body mass (obesity) and high androgen levels, APN supplementation during the first trimester of pregnancy is an effective preventive strategy to correct the metabolic disorders of female offspring in adulthood. However, adverse drug reactions and the use-value of this program in clinical practice still need to be further explored.

\section{Abbreviations}

AF: Antral follicle; Akt/PKB: Protein kinase B; AMH: Anti mullerian hormone; AMPK: Adenosine monophosphate activated protein kinase;

APN: Adiponectin; CAMKK2: Calcium/calmodulin dependent protein kinase kinase2; CF: Cystic follicle; CL: Corpora lutea; COX2: Cyclooxygenase-2 protein; DHEA: Dehydroepiandrosterone; FBG: Fasting blood glucose; GC: Granulosa cell; GSK3ß: Glycogen synthase kinase 3ß; HCG: Human chorionic gonadotropin; HDL-C: High density lipoprotein cholesterol; HOMAIR: Homeostasis model assessment-insulin resistance; INS: Insulin; I.p: Intraperitoneally; IPGTT: Intraperitoneally glucose tolerance test; IPIT T: Intraperitoneally insulin tolerance test; IRS-1: Insulin receptor substrate-1; LDL-C: Low density lipoprotein cholesterol; PCOS: Polycystic ovary syndrome; PF: Preovulatory follicle; PI3K: Phosphatidylinositol 3-kinase; PMSG: pregnant mare serum gonadotropin; PPARy: Peroxisome proliferator-activated receptors $\gamma$; qRT- PCR: Quantitative real- time PCR; SHBG: Sex hormonebinding globulin; S6K1: Ribosomal protein S6 kinase 1; StAR: Steroidogenic acute regulatory protein; T: Testosterone; TC: Total cholesterol;

TG: Triglycerides

\section{Acknowledgements}

Not applicable.

\section{Authors' contributions}

MZ carried out all of the experiments. GL and DX have made substantial contributions to conception and design of study. MZ and GL wrote the manuscript. WZ and JL performed experiments. MT, YY and $\mathrm{CH}$ analyzed data. YW has been involved in acquisition of data, drafting the manuscript, and revising it critically for important intellectual content. All authors read and approved the final manuscript.

\section{Funding}

Guangzhou Science and Technology Projects, Grant/Award Number: NO.201707010265.

\section{Availability of data and materials}

All the data is contained in the manuscript.

\section{Ethics approval and consent to participate}

The experiments were conducted in accordance with the Regulation of National Experimental Animal Welfare Ethics in China. The ethical approval was granted by the Jinan University Experimental Animal Ethics Committee (Project number IACUC-20190125-08).

\section{Consent for publication}

Not applicable.

\section{Competing interests}

The authors declare that they have no competing interests.

\section{Author details}

'Department of Reproductive Medicine, The First Affiliated Hospital, Jinan University, 601 West Huangpu Avenue, 510000 Guangzhou, People's Republic of China. ${ }^{2}$ The Second Hospital, University of South China, 421001 Hengyang, People's Republic of China. ${ }^{3}$ Department of Obstetrics and Gynecology, The Second People's Hospital of Yueyang, 414000 Yueyang, People's Republic of China.

Received: 31 July 2020 Accepted: 11 December 2020

Published online: 18 January 2021

\section{References}

1. Jayasena CN, Franks S. The management of patients with polycystic ovary syndrome. Nat Rev Endocrinol. 2014;10(10):624-36.

2. Teede HJ, Misso ML, Costello MF, et al. International PCOS Network Recommendations from the international evidence-based guideline for the assessment and management of polycystic ovary syndrome. Fertil Steril. 2018;110(3):364-79.

3. Escobar-Morreale HF. Polycystic ovary syndrome: definition, aetiology, diagnosis and treatment. Nat Rev Endocrinol. 2018;14(5):270-84.

4. Norman RJ, Dewailly D, Legro RS, et al. Polycystic ovary syndrome. Lancet. 2007:370:685-97.

5. Fraissinet $A$, Robin $G$, et al. Use of the serum anti-Müllerian hormone assay as a surrogate for polycystic ovarian morphology: impact on diagnosis and phenotypic classification of polycystic ovary syndrome. Hum Reprod. 2017; 32(8):1716-22

6. Kataoka J, Larsson I, et al. Prevalence of polycystic ovary syndrome in women with severe obesity - effects of a structured weight loss programme. Clin Endocrinol (Oxf). 2019;91(6):750-8.

7. De Melo AS, Dias SV, Cavalli RC, et al. Pathogenesis of polycystic ovary syndrome: multifactorial assessment from the foetal stage to menopause. Reproduction. 2015;150(1):R11-24.

8. Sir-Petermann T, Maliqueo M, Codner E, et al. Early metabolic derangements in daughters of women with polycystic ovary syndrome. J Clin Endocrinol Metab. 2007;92(12):4637-42.

9. McAllister JM, Legro RS, Modi BP, et al. Functional genomics of PCOS: from GW AS to molecular mechanisms. Trends in endocrinology metabolism. 2015;26(3):118-24

10. Yan XN, Yuan C, Zhao NN, et al. Prenatal Androgen Excess Enhances Stimulation of the GNRH Pulse in Pubertal Female Rats. The Journal of Endocrinology. 2014;222(1):73-85.

11. Roland AV, Moenter SM. Prenatal androgenization of female mice programs an increase in firing activity of gonadotropin-releasing hormone (GnRH) neurons that is reversed by metformin treatment in adulthood. Endocrinology. 2011;152(2):618-28.

12. Caldwell AS, Eid S, Kay CR, et al. Haplosufficient genomic androgen receptor signaling is adequate to protect female mice from induction of polycystic ovary syndrome features by prenatal hyperandrogenization. Endocrinology. 2015;156(4):1441-52.

13. Tata B, Mimouni NEH, Barbotin AL, et al. Elevated prenatal anti-Mullerian hormone reprograms the fetus and induces polycystic ovary syndrome in adulthood. Nat Med. 2018;24(6):834-46.

14. Tanabe H, Fujii Y, Okadaiwabu M, et al. Crystal structures of the human adiponectin receptors. Nature. 2015;520(7547):312-6.

15. Goldfine $A B$, Kahn $C R$. Adiponectin: linking the fat cell to insulin sensitivity. Lancet. 2003;362(9394):1431-2.

16. Dumesic DA, Lobo RA. Cancer risk and PCOS. Steroids. 2013;78(8):782-5.

17. Parida S, Siddharth S, Sharma D, et al. Adiponectin, Obesity, and Cancer: Clash of the Bigwigs in Health and Disease. Int J Mol Sci. 2019;20(10):2519.

18. Wild RA, Carmina E, Diamantikandarakis E, et al. Assessment of cardiovascular risk and prevention of cardiovascular disease in women with the polycystic ovary syndrome: a consensus statement by the Androgen Excess and Polycystic Ovary Syndrome (AE-PCOS) Society. J Clin Endocrinol Metab. 2010;95(5):2038-49.

19. Spranger J, Kroke A, Mohlig M, et al. Adiponectin and protection against type 2 diabetes mellitus. Lancet. 2003;361(9353):226-8.

20. Yamauchi T, Nio Y, Maki T, et al. Targeted disruption of AdipoR1 and AdipoR2 causes abrogation of adiponectin binding and metabolic actions. Nat Med. 2007;13(3):332-9.

21. Herzig S, Shaw RJ. AMPK: Guardian of Metabolism and Mitochondrial Homeostasis. Nat Rev Mol Cell Biol. 2018;19(2):121-35. 
22. Chopra I, Li HF, Wang H, et al. Phosphorylation of the insulin receptor by AMP-activated protein kinase (AMPK) promotes ligand-independent activation of the insulin signaling pathway in rodent muscle. Diabetologia. 2012;55(3):783-94.

23. Iwabu M, Yamauchi T, Okada-Iwabu M, et al. Adiponectin and AdipoR1 regulate PGC-1alpha and mitochon- dria by $\mathrm{Ca}(2+)$ and AMPK/SIRT1. Nature. 2010;464(7293):1313-9.

24. Yamauchi T, Kamon J, Minokoshi Y, et al. Adiponectin stimulates glucose utilization and fatty-acid oxidation by activating AMP-activated protein kinase. Nature Medicine. 2002;8(11):1288-95.

25. Horike N, Sakoda H, Kushiyama A, et al. AMP-activated protein kinase activation increases phosphorylation of glycogen synthase kinase 3beta and thereby reduces CAMP-responsive element transcriptional activity and phosphoenolpyruvate carboxykinase $\mathrm{C}$ gene expression in the liver. J Biol Chem. 2008;283(49):33902-10.

26. Iliodromiti S, Sassarini J, Kelsey TW, et al. Accuracy of circulating adiponectin for predicting gestational diabetes: a sys-tematic review and meta-analysis. Diabetologia. 2016;59:692-9.

27. Retnakaran R, Ye C, Kramer CK, et al. Evaluation of circulating determinants of beta-cell function in women with and without gestational diabetes. J Clin Endocrinol Metab. 2016;101(7):2683-91.

28. Li S, Shin HJ, Ding EL, et al. Adiponectin levels and risk of type 2 diabetes: a systematic review and meta-analysis. JAMA. 2009;302(2):179-88.

29. Qiao L, Wattez J-S, Lee S, et al. Adiponectin deficiency impairs maternal metabolic adaptation to pregnancy in mice. Diabetes. 2017;66(5):1126-35.

30. Dowling AR, Nedorezov LB, Qiu X, et al. Genetic Factors Modulate the Impact of Pubertal Androgen Excess on Insulin Sensitivity and Fertility. PLoS ONE, 2013, 8(11).

31. Hao Lai X, Jia Q. Yu, et al. High-fat diet induces significant metabolic disorders in a mouse model of polycystic ovary syndrome. Biol Reprod,2014, 91(5):127.

32. Singh A, Bora P, Krishna A. Systemic adiponectin treatment reverses polycystic ovarysyndrome-like features in an animal model. Reprod Fertil Dev. 2018;Mar 30(4):571-84.

33. Cimino I, Casoni F, Liu XH, et al. Novel role for anti-Mullerian hormone in the regulation of $\mathrm{GnRH}$ neuron excitability and hormone secretion. Nature communications. 2016;7(1):10055.

34. Hanley AJG, Williams K, Stern MP, Haffner SM. Homeostasis Model Assessment of Insulin Resistance in Relation to the Incidence of Cardiovascular Disease The San Antonio Heart Study. Diabetes Care. 2002; 25:1177-84.

35. Palomba S, Marotta R, di Cello A, et al. Pervasive developmental disorders in children of hyperandrogenic women with polycystic ovary syndrome: a longitudinal case-control study. Clin Endocrinol. 2012;77(6): 898-904.

36. Franik G, Maksym M, Owczarek AJ, et al. Estradiol/testosterone and estradiol/androstenedione indexes and nutritional status in PCOS women A pilot study. Eur J Obstet Gynecol Reprod Biol. 2019;242:166-9.

37. Chang RJ. The reproductive phenotype in polycystic ovary syndrome. Nat Clin Pract Endocrinol Metab. 2007;3:688-95.

38. Shrestha N, Ezechukwu HC, et al. Developmental programming of peripheral diseases in offspring exposed to maternal obesity during pregnancy. Am J Physiol Regul Integr Comp Physiol. 2020;10:1152.

39. De Leo V, Musacchio MC, Cappelli V, et al. Genetic, hormonal and metabolic aspects of PCOS: an update. Reprod Biol Endocrinol. 2016;14(1):38.

40. Anderson AD, Solorzano CMB, Mccartney CR, et al. Childhood obesity and its impact on the development of adolescent PCOS. Seminars Reproductive Med. 2014;32(3):202-13.

41. Xita N, Tsatsoulis A. Fetal origins of the metabolic syndrome. Ann N Y Acad Sci. 2010;1205(1):148-55.

42. Palomba S, deWilde MA, Falbo A, et al. Reply: Polycystic ovary syndrome and pregnancy. Hum Reprod Update. 2016;22(2):278-9.

43. Sobrevia $L$, Valero $P$, et al. Mitochondrial dysfunction in the fetoplacental unit in gestational diabetes mellitus. Biochim Biophys Acta Mol Basis Dis. 2020; 165948.

44. Kuscu NK, Var A. Oxidative stress but not endothelial dysfunction exists in nonobese, young group of patients with polycystic ovary syndrome. Acta Obstet Gynecol Scand. 2009;88(5):612-7.

45. Amalfi S, Amalfi S, Velez LM, et al. Prenatal Hyperandrogenization Induces Metabolic and Endocrine Alterations Which Depend on the Levels of Testosterone Exposure. PLoS One. 2012;7(5):e37658.
46. Xenopoulos $\mathrm{P}$, Kang M, et al. Heterogeneities in Nanog Expression Drive Stable Commitment to Pluripotency in the Mouse Blastocyst. Cell Rep. 2015; 10(9):1508-20.

47. Bolnick A, Abdulhasan M, et al. Commonly used fertility drugs, a diet supplement, and stress force AMPK-dependent block of stemness and development in cultured mammalian embryos. J Assist Reprod Genet. 2016; 33(8):1027-39.

\section{Publisher's Note}

Springer Nature remains neutral with regard to jurisdictional claims in published maps and institutional affiliations.
Ready to submit your research? Choose BMC and benefit from:

- fast, convenient online submission

- thorough peer review by experienced researchers in your field

- rapid publication on acceptance

- support for research data, including large and complex data types

- gold Open Access which fosters wider collaboration and increased citations

- maximum visibility for your research: over $100 \mathrm{M}$ website views per year

At BMC, research is always in progress.

Learn more biomedcentral.com/submissions 